В. Н. Демидов ${ }^{1}$, С. М. Сухаржевскийㄹ, С. В. Пастон ${ }^{2}$, А. В. Зинченко ${ }^{3}$, Л. Н. Веденеева ${ }^{4}$, Т. Б. Пахомова ${ }^{3}$

\title{
ТЕРМОИНДУЦИРОВАННЫЕ $S_{0} \rightarrow T$-ПЕРЕХОДЫ В ЭЛЕКТРОН-ИЗБЫТОЧНЫХ 1,10-ФЕНАНТРОЦИАНИНАХ Zn(II), КОМПЛЕКСОНАХ ДНК, СОДЕРЖАЩИХ ДИГИДРО-1,10-ФЕНАНТРОЛИНОВЫЕ ЦИКЛЫ, РОДСТВЕННЫЕ NADH*
}

1 Концерн «Рro-Brite», Российская Федерация, 192289, Санкт-Петербург, ул. Софийская, 93

2 Санкт-Петербургский государственный университет,

Российская Федерация, 199034, Санкт-Петербург, Университетская наб., 7-9

3 Санкт-Петербургский государственный технологический институт (технический университет), Российская федерация, 190013, Санкт-Петербург, Московский пр., 26

4 Пермский национальный исследовательский политехнический университет, Березниковский филиал, Российская федерация, 618400, Березники, ул. Юбилейная, 99

С помощью спектроскопии ЭПР изучено проявление парамагнитных свойств для электрон-избыточных 1,10-фенантроцианиновых комплексов $\operatorname{Zn}(\mathrm{II})$ : [(phen) $2 \mathrm{Zn}(\mu$-phencyanine) $\left.\mathrm{Zn}(\text { phen })_{2}\right](\mathrm{OAc})_{4} \quad(\mathrm{Zn} 1), \quad\left[(\text { phen })_{2} \mathrm{Zn}\left(\mu\right.\right.$-phencyanine $\left.\left.{ }^{-}\right) \mathrm{Zn}(\mathrm{phen})_{2}\right](\mathrm{OAc})_{3} \quad(\mathrm{Zn} 2), \quad(\mathrm{phen}) \mathrm{Zn}(\mu-$ phencyanine $\left.^{-}\right) \mathrm{Zn}($ phen $)(\mathrm{OAc})_{3}(\mathrm{Zn} 3),($ en $) \mathrm{Zn}\left(\mu\right.$-phencyanine $\left.{ }^{-}\right) \mathrm{Zn}(\mathrm{en})(\mathrm{OAc})_{3}(\mathrm{Zn} 4)$ и $\mathrm{Zn}(\mu$-phencyanine $\left.^{-}\right) \operatorname{Zn}(\mathrm{OAc})_{3}$ (Zn5) (phen - 1,10-фенантролин, en - этилендиамин, ОАс ${ }^{-}$- ацетатный ион, $\mu$-phencyanine и $\mu$-phencyanine ${ }^{-}$- мостиковые 1,10-фенантроцианины, дигидро-би1,10-фенантролины), комплексонов ДНK, содержащих родственные NADH дигидро-1,10-фенантролиновые циклы. Соединения Zn1-Zn5 получены с использованием методологии металл-опосредуемого недегидрогенативного $\mathrm{CH}-\mathrm{CH}-$ сочетания координированного 1,10-фенантролина в ионных расплавах соответствующих комплексов-предшественников. В ЭПР спектрах полученных 1,10-фенантроцианинов $\mathrm{Zn}(\mathrm{II})$ обнаружены сигналы в области значений g-фактора 2,0018-2,0031 с неожиданно высокой интенсивностью. Установлено аномальное увеличение интенсивности линий ЭПР с ростом температуры от 77 до $450 \mathrm{~K}$. Появление парамагнетизма для этих соединений соотнесено с их электрон-избыточной природой и интерпретировано как результат термоиндуцированных $S_{0} \rightarrow T$-переходов, которые можно связать со структурными фазовыми превращениями. Библиогр. 21 назв. Ил. 1. Табл. 1.

Ключевые слова: электрон-избыточные 1,10-фенантроцианины $\mathrm{Zn}(\mathrm{II})$, спектроскопия ЭПР, термоиндуцированные $S_{0} \rightarrow T$-переходы, термически доступные триплетные состояния.

Для цитирования: Демидов В. Н., Сухаржевский С. М., Пастон С. В., Зинченко А. В., Веденеева Л.Н., Пахомова Т. Б. Термоиндуцированные $S_{0} \rightarrow T$-переходы в электрон-избыточных 1,10-фенантроцианинах $\mathrm{Zn}(\mathrm{II})$, комплексонах ДНК, содержащих дигидро-1,10фенантролиновые циклы, родственные NADH // Вестник СПбГУ. Физика и химия. 2017. T. 4 (62). Вып. 2. C. 138-145. DOI: 10.21638/11701/spbu04.2017.203

* По материалам IV конференции «Современные проблемы молекулярной биофизики», посвящённой 50-летию специализации «Молекулярная биофизика» на физическом факультете и 105-летию со дня рождения профессора Э. В. Фрисман, 14-15 июня 2016 г., СПбГУ, Санкт-Петербург, Россия, URL: http://molbioph.niif.spbu.ru/conference.

(C) Санкт-Петербургский государственный университет, 2017 
V.N.Demidov ${ }^{1}$, S.M.Sukharzhevsky ${ }^{2}$, S.V.Paston ${ }^{2}$, A. V. Zinchenko ${ }^{3}$, L. N. Vedeneeva ${ }^{4}$, T. B. Pakhomova ${ }^{3}$

\section{THERMOINDUCED $S_{0} \rightarrow T$-TRANSITIONS IN ELECTRON-RICH Zn(II) 1,10-PHENANTHROCYANINES, DNA COMPLEXONES CONTAINING DIHYDRO-1,10-PHENANTHROLINE CYCLES RELATED TO NADH}

1 Concern "Pro-Brite", 93, ul. Sofiyskaya, St. Petersburg, 192289, Russian Federation

2 St. Petersburg State University,

7-9, Universitetskaya nab., St. Petersburg, 199034, Russian Federation

3 St. Petersburg State Technological Institute (Technical University), 26, Moskovsky pr., St. Petersburg, 190013, Russian Federation

4 Permsky National Research Polytechnical University, 99, Yubileynaya ul., Berezniky, 618400, Russian Federation

Manifestation of paramagnetic properties for electron-rich $\mathrm{Zn}$ (II) 1,10-phenanthrocyanine: $\left[(\text { phen })_{2} \mathrm{Zn}(\mu\right.$-phencyanine $\left.) \mathrm{Zn}(\text { phen })_{2}\right](\mathrm{OAc})_{4} \quad(\mathrm{Zn} 1), \quad\left[(\text { phen })_{2} \mathrm{Zn}\left(\mu\right.\right.$-phencyanine $\left.\left.{ }^{-}\right) \mathrm{Zn}(\text { phen })_{2}\right]$ (OAc) $_{3}(\mathrm{Zn} 2)$, (phen)Zn( $\mu$-phencyanine $\left.{ }^{-}\right) \mathrm{Zn}($ phen $)(\mathrm{OAc})_{3}(\mathrm{Zn} 3),(\mathrm{en}) \mathrm{Zn}\left(\mu\right.$-phencyanine $\left.{ }^{-}\right) \mathrm{Zn}(\mathrm{en})$ $(\mathrm{OAc})_{3}(\mathrm{Zn} 4)$ and $\mathrm{Zn}\left(\mu\right.$-phencyanine $\left.{ }^{-}\right) \mathrm{Zn}(\mathrm{OAc})_{3}(\mathrm{Zn} 5)$ (phen - 1,10-phenanthroline, en - ethylenediamine, $\mathrm{OAc}^{-}$- acetate ion, $\mu$-phencyanine and $\mu$-phencyanine ${ }^{-}$-bridge 1,10 -phenanthrocyanines, dihydro-bi-1,10-phenanthrolines), DNA complexones containing related to NADH dihydro-1,10-phenanthroline cycles were investigated by ESR spectroscopy. Compounds of Zn1-Zn5 are derived using methodologies of metal-mediated nondehydrogenative $\mathrm{CH}-\mathrm{CH}$ coupling of coordinated 1,10-phenanthroline in ionic melts of respective complexes-precursors. In ESR spectra of derived 1,10-phenanthrocyanines of $\mathrm{Zn}(\mathrm{II})$ signals are detected in the values area of g-factor 2.0018-2.0031 with unexpectedly high intensity. Abnormal increase of intensity of ESR line halfwidth with growth of temperature from 77 to $450 \mathrm{~K}$ were found. Appearance of paramagnetism for these compounds was correlated with their electron-rich nature and as result of thermoinduced $S_{0} \rightarrow T$-transitions were interpreted that can be associated with structural phase transitions. Refs 21. Figs 1. Tables 1.

Keywords: electron-rich 1,10-phenanthrocyanine of $\mathrm{Zn}(\mathrm{II})$, ESR spectroscopy, thermoinduced $S_{0} \rightarrow T$-transitions, thermally accessible triplet states.

For citation: Demidov V. N., Sukharzhevsky S. M., Paston S V., Zinchenko A. V., Vedeneeva L. N., Pakhomova T.B. Thermoinduced $S_{0} \rightarrow T$-transitions in electron-rich $\mathrm{Zn}(\mathrm{II}) 1,10-$ phenanthrocyanines, DNA complexones containing dihydro-1,10-phenanthroline cycles related to NADH. Vestnik SPbSU. Physics and Chemistry. 2017, vol. 4 (62), issue 2, pp. 138-145. DOI: $10.21638 / 11701 / \mathrm{spbu04.2017.203}$

Введение. Координационные соединения цинка с 1,10-фенантролинами и их производные способны проявлять противовирусные, антибактериальные и противогрибковые свойства [1-5]. В работах [6-14] исследуются механизмы связывания 1,10-фенантролиновых комплексов цинка с макромолекулами ДНК, а в работах $[9,10,12]$ исследуется также их способность инициировать расщепление ДНК (проявлять нуклеазную активность). Для комплексов этого класса обнаружено выраженное противоопухолевое действие $[8,11,13,15]$. В качестве комплексонов ДНК и противоопухолевых агентов интенсивно исследуются смешаннолигандные 1,10-фенантролиновые соединения цинка $[2,9-14]$. В связи с малой токсичностью ионов $\mathrm{Zn}^{2+}$, их редокс-индифферентностью и участием в функционировании природных металлоферментов эти комплексы представляют особый интерес, как потенциальные лекарственные агенты. К проявлению подобных свойств склонны и электрон-избыточные пурпурные 1,10-фенантроцианины $\mathrm{Zn}(\mathrm{II})$, содержащие редокс-активные дигидро-1,10-фенантролиновые циклы, родственные NADH. Для некоторых из них ранее была обнаружена склонность 
к комплексообразованию с молекулами ДНК [16]. Принимая во внимание вышесказанное, в работе представлены результаты изучения термоиндуцированных $S_{0} \rightarrow T$-переходов методом ЭПР.

Материалы и методы. В качестве объектов исследования выбраны биядерные электрон-избыточные мостиковые 1,10-фенантроцианины Zn(II): [(phen $)_{2} \mathrm{Zn}(\mu$-phencyanine $\left.) \mathrm{Zn}(\text { phen })_{2}\right](\mathrm{OAc})_{4}-(\mathrm{Zn} 1), \quad\left[(\text { phen })_{2} \mathrm{Zn}\left(\mu\right.\right.$-phencyanine $\left.\left.^{-}\right) \mathrm{Zn}(\text { phen })_{2}\right](\mathrm{OAc})_{3}-(\mathrm{Zn} 2)$, (phen)Zn( $\mu$-phencyanine $\left.{ }^{-}\right) \operatorname{Zn}($ phen $)(\mathrm{OAc})_{3}-\quad(\mathrm{Zn} 3), \quad($ en $) \mathrm{Zn}\left(\mu\right.$-phencyanine $\left.{ }^{-}\right) \mathrm{Zn}($ en $)$ $(\mathrm{OAc})_{3}-(\mathrm{Zn} 4)$ и $\mathrm{Zn}\left(\mu\right.$-phencyanine $\left.{ }^{-}\right) \mathrm{Zn}(\mathrm{OAc})_{3}-(\mathrm{Zn} 5)$. Здесь phen - 1,10-фенантролин, en - этилендиамин, $\mathrm{OAc}^{-}$- ацетатный ион, $\mu$-phencyanine и $\mu$-phencyanine ${ }^{-}$- мостиковые 1,10-фенантроцианины, дигидро-би-1,10-фенантролины. Комплексы получены по методологии металл-опосредуемого недегидрогенативного $\mathrm{CH}-\mathrm{CH}$-сочетания 1,10-фенантролина в ионных расплавах соответствующих 1,10-фенантролиновых ацетатов $\mathrm{Zn}(\mathrm{II})$ [17]: [Zn(phen $\left.)_{3}\right](\mathrm{OAc})_{2}, \mathrm{Zn}(\mathrm{phen})_{2}(\mathrm{OAc})_{2}$ и $\mathrm{Zn}($ phen $)(\mathrm{OAc})_{2}$. Во время измерения спектров образцы были открыты для доступа атмосферного воздуха.

Результаты и обсуждение. Исходя из общих соображений комплексы 1,10-фенантроцианинов Zn(II) (образцы Zn1-Zn5) при температурах $300 \mathrm{~K}$ и ниже не должны проявлять парамагнитные свойства. Однако в пурпурных разновидностях этих образцов как в стеклообразном состоянии, так и в растворах $\mathrm{CHCl}_{3}$ при температуре $300 \mathrm{~K}$ наблюдается спектр ЭПР, состоящий из одной (Zn1-Zn4) (см. рисунок) или двух линий (Zn5).

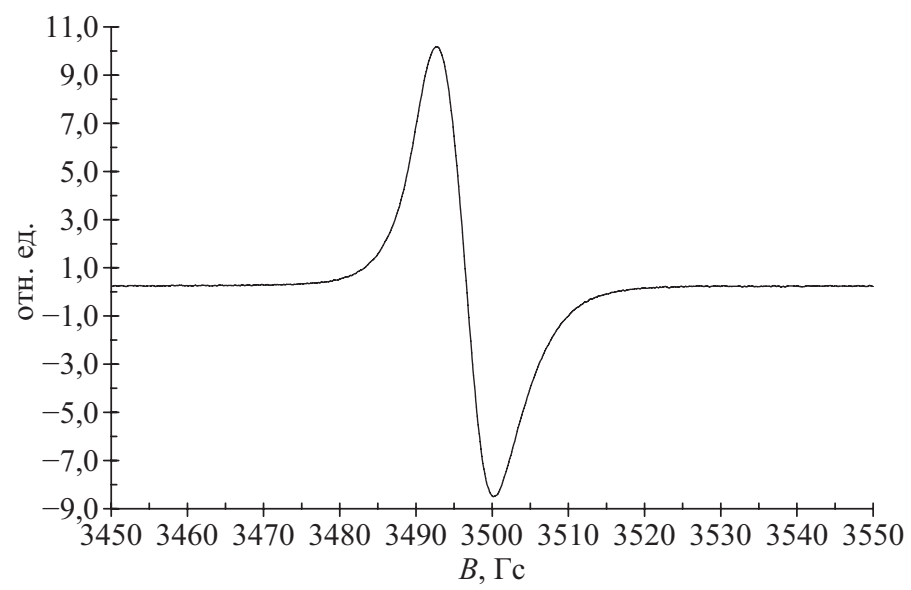

Спектр ЭПР образца Zn2 в стеклообразном состоянии при $T=300 \mathrm{~K}$

Значения параметров линий представлены в таблице. Из таблицы хорошо видно, что все линии ЭПР характеризуются слабой анизотропией g-фактора. Также необходимо отметить что, наибольшей интенсивностью характеризуются линии с g-факторами бо́льшими, чем g-фактор свободного электрона 2,0023. Сравнивая интегральные интенсивности низкополевого и высокополевого сигналов, следует отметить, что для последнего она примерно в 65 раз меньше.

Для образцов Zn2 и Zn3 анизотропия заметна меньше, а в образце Zn2 наблюдается практически изотропный сигнал. Проведённый анализ формы линии позволяет сделать предположение о её лоренцевой природе. Изотропность сигнала указывает, прежде всего, на высокую симметрию распределения спиновой плотности на мостиковом лиганде, а его лоренцева форма - на участие этого спина в прямых обмен- 
ных взаимодействиях. Высокая степень делокализации спина в пределах мостикового 1,10-фенантроцианинового лиганда может объяснить отсутствие разрешённых суперсверхтонких расщеплений ЭПР-линий. Подобный сигнал наблюдается в спектрах ЭПР анион-радикалов замещённых 1,10-фенантролинов при условии существенного $\pi$ $\pi$-сопряжения функциональных групп заместителей с гетероциклическим кольцом [18]. Кроме того отсутствие суперсверхтонкой структуры может быть результатом спинобменных взаимодействий в бирадикалах, как это наблюдается для димерных радикалов виологенов [19].

Параметры спектров ЭПР стеклообразных 1,10-фенантроцианинов Zn(II)

\begin{tabular}{c|c|c}
\hline Комплекс & $g_{\|}$ & $g_{\perp}$ \\
\hline Zn1 & 2,0023 & 2,0045 \\
\hline Zn2 & $2,0031^{*}$ & - \\
\hline Zn3 & $2,0027^{*}$ & - \\
\hline Zn4 & 2,0018 & 2,0040 \\
\hline \multirow{2}{*}{ Zn5 } & 2,0023 & 2,0045 \\
& 1,9660 & 1,9593 \\
\hline
\end{tabular}

* - для почти изотропных сигналов приведены эффективные значения $g_{\text {эф. }}$.

Проведённые температурные измерения показывают, что с повышением температуры от 110 до 413 K (температуры плавления комплекса) интенсивность сигналов (концентрация) радикальных центров 1,10-фенантроцианинов $\mathrm{Zn}(\mathrm{II})$ прогрессивно растёт. Это противоречит закону Кюри для простых магнитноразбавленных парамагнетиков. Ширина линии основного сигнала с увеличением температуры почти не меняется (наблюдается её незначительный рост).

Своим происхождением ЭПР-сигналы 1,10-фенантроцианинов $\mathrm{Zn}(\mathrm{II})$, скорее всего, обязаны наличию в структуре соединений электрон-избыточных мостиковых лигандов $\mu$-phencyanine. При этом для расплавов возможно установление равновесия, а для стеклообразной фазы - псевдоравновесия (аналог структурных фазовых переходов второго рода), сопряжённого с протонным переносом между исходным диамагнитным (D) и конечным парамагнитным (P) состояниями, как это показано на схеме. При таком механизме, для температур от комнатной и ниже равновесие сдвинуто в сторону диамагнитного состояния, а при повышении температуры, вплоть до температур плавления соединения (430-450 K) оно смещено в сторону формирования триплетных бирадикалов. В окрестности точек плавления наблюдается резкое увеличение интенсивности ЭПР-сигналов. Поэтому, в соответствии с термодинамическим принципом Ле-Шателье-Брауна, можно говорить о том, что процесс образования бирадикалов эндотермический, и переход $\mathrm{D}(S) \rightarrow \mathrm{P}(T)$ осуществляется как термоиндуцированный.

Центральная связь С-C в 1,10-фенантроцианинах, соединяющая два 1,10-фенантролиновых структурных фрагмента имеет кратность (в первом приближении) около 1,5. Заторможенный поворот вокруг неё, по-видимому, может быть термически активирован, что приведёт к формированию трилетных бирадикалов. Согласно DFT-расчётам

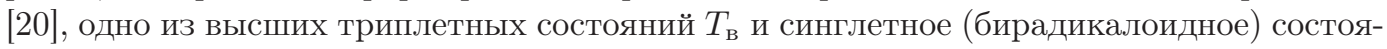
ние $S_{1} 1,10$-фенантроцианинов энергетически близки. Полученные в настоящей работе результаты указывают на то, что основное синглетное состояние $S_{0}$ 1,10-фенантроциа-

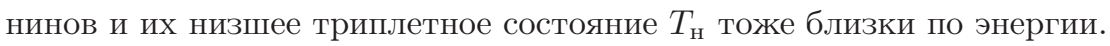

Объяснение природы ЭПР-сигналов 1,10-фенантроцианинов как результат термического заселения низшего триплетного состояния $T_{\mathrm{H}}$ из основного состояния $S_{0}(h v \sim k T)$ 
в процессе термического электронного возбуждения, связанного с гомолизацией, ингибированием двоесвязности и переходом в бирадикальное состояние (см. схему) согласуется с подходами, развиваемыми в [21]. Согласно им, для донорно-акцепторных систем возможно существование термически достижимых электронно-возбуждённых состояний, в особенности, если переход в одно из таких состояний сопровождается протонным переносом. При термическом переходе 1,10-фенантроцианинов $\mathrm{Zn}(\mathrm{II})$ из диамагнитной формы в парамагнитную как раз и реализуется подобный протонный перенос (см. схемy).

$$
\begin{gathered}
\pi-\pi-(\text { phen })=(\text { dihydro })-(\text { phen }) /(\text { dihydro })-(\text { phen })=(\text { phen }) \quad p, p \text { - }\left(\text { phen }^{-\cdot}\right)-\left(\text { phen }^{-\cdot}\right) /+2 \mathrm{H}^{+} \\
\mathrm{D}: \mathrm{Zn}^{2+}(\mu \text {-phencyanine }) \mathrm{Zn}^{2+}(S)^{+} Q(+h v) \rightarrow \mathrm{P}: \mathrm{Zn}^{2+}(\mu \text {-phencyanine })^{*} \mathrm{Zn}^{2+}(T) \\
\text { диамагнетик бирадикал, парамагнетик }
\end{gathered}
$$

Схема возникновения бирадикальных парамагнитных форм

\section{Выводы.}

1. В спектрах ЭПР электрон-избыточных биядерных пурпурных 1,10-фенантроцианиновых комплексов $\mathrm{Zn}(\mathrm{II})$ обнаружены относительно интенсивные сигналы с g-фактором 2,0018-2,0031. Измеренные нами ЭПР-сигналы характеризуются слабой анизотропией, а для комплекса Zn2 сигнал является практически изотропным. С ростом температуры от 100 до $450 \mathrm{~K}$ интенсивность сигналов в спектрах ЭПР растёт, особенно резко - вблизи температур плавления соединений (430-450 K).

2. Появление в спектрах ЭПР относительно интенсивных сигналов следует связать с мостиковыми хромофорными электрон-избыточными 1,10-фенантроцианиновыми лигандами, способными переходить из обычной диамагнитной синглетной формы (основное состояние) в форму термически доступных парамагнитных триплетных бирадикалов.

$$
* * *
$$

ЭПР-исследования комплексов цинка были проведены в Ресурсном центре «Магнитно-резонансные методы исследования» Научного парка СПбГУ на спектрометре ELEXSYS-E580 (X-BAND).

\section{Литература}

1. Oladipo M.A., Olaoye O. J. Antimicrobial, DNA cleavage and antitumoral properties of some transition metal complexes of 1,10-phenanthroline and 2, 2'-bi-pyridine: A review // Int. J. Res. Pharm. Biomed. Sci. 2013. Vol. 4, N 4. P. 1160-1171.

2. Mahalakshmi R., Raman N. A therapeutic journey of mixed ligand complexes containing 1,10-phenanthroline derivatives: a review // Int. J. Curr. Pharm. Res. 2016. Vol. 8, N 3. P. 1-6.

3. Shulman A., White D. O. Virostatic activity of 1,10-phenanthroline transition metal chelates: A structure-activity analysis // Chem.-Biol. Interactions. 1973. Vol. 6. P. 407-413.

4. Dwyer F. P., Reid I. K., Shulman A., Laycock G. M., Dixson S. The biological actions of 1,10-phenanthroline and 2, 2'-bipyridine hydrochlorides, quaternary salts and metal chelates and related compounds. 1. Bacteriostatic action on selected gram-positive, gram-negative and acid-fast bacteria // Austral. J. Exper. Biol. Med. Sci. 1969. Vol. 47. P. 203-218.

5. Shulman A., Cade G., Dumble L., Laycock G. M. The lethal action of 1,10-phenanthroline transition metal chelates and related compounds on dermatophytes and Candida albicans // Arzneimittelforschung. 1972. Bd. 22, N 1. S. 154-158. 
6. Barton J. K., Dannenberg J. J., Raphael A. L. Enantiomeric selectivity in binding tris-(phenanthroline)zinc(II) to DNA // J. Amer. Chem. Soc. 1982. Vol. 104, N 18. P. 4967-4969.

7. Shahabadi N., Mohammadi S. Synthesis, characterization and DNA interaction studies of a new Zn(II) complex containing different dinitrogen aromatic ligands // Bioinorg. Chem. Appl. 2012. Vol. 2012. 571913.

8. Anjomshoa M., Torkzadeh-Mahani M., Shakeri M. The Zn(II) nanocomplex: sonochemical synthesis, characterization, DNA- and BSA-binding, cell imaging, and cytotoxicity against the human carcinoma cell lines // J. Fluorescence. 2016. Vol. 26, N 3. P. 1007-1020.

9. Yuan Cai-Xia, Wei Yi-Bin, Yang Pin. DNA-binding and cleavage studies of zinc(II) mixed-polypyridyl complex // Chinese J. Chem. 2006. Vol. 24, N 8. P. 1006-1012.

10. Hoi-Ling Seng, Sze-Tin Von, Kong-Wai Tan, Mohd Jamil Maah, Seik-Weng Ng, Raja Noor Zaliha Raja Abd Rahman, Ignez Caracelli, Chew-Hee $N g$. Crystal structure, DNA binding studies, nucleolytic property and topoisomerase I inhibition of zinc complex with 1,10-phenanthroline and 3-methyl-picolinic acid // BioMetals. 2010. Vol. 23. P. 99-118.

11. Von S.-T., SengH.-L., LeeH.-B., NgS.-W., Kitamura Y., ChikiraM., NgC.-H. DNA molecular recognition and cellular selectivity of anticancer metal(II) complexes of ethylene- diaminediacetate and phenanthroline: multiple targets // J. Biolog. Inorg. Chem. 2012. Vol. 17, N 1. P. 57-69.

12. Seng H.-L., OngH.-K.A., Rahman R. N.Z.R. Abd., Yamin B. M., TiekinkE. R. T., Tan K. W., Maah M. J., Caracelli I., Ng C.-H. Factors affecting nucleolytic efficiency of some ternary metal complexes with DNA binding and recognition domains. Crystal and molecular structure of $\mathrm{Zn}(\mathrm{phen})$ (edda) // J. Inorg. Biochem. 2008. Vol. 102, N 11. P. 1997-2011.

13. Qin Wang, Min Huang, Yu Huang, Jin-Sheng Zhang, Gui-Feng Zhou, Ren-Quan Zeng, Xin-Bin Yang. Synthesis, characterization, DNA interaction, and antitumor activities of mixed- ligand metal complexes of kaempferol and 1,10-phenanthroline/2,2'-bipyridine // Med. Chem. Res. 2014. Vol. 23 , N 5. P. 2659-2666.

14. Gouvea L. R., Martins D. A., BatistaD. G. J., Soeiro M. N. M. C., Louro S. R. W., Barbeira P. J. S., Teixeira L. R. Norfloxacin Zn(II)-based complexes: acid base ionization constant determination, DNA and albumin binding properties and the biological effect against Trypanosoma cruzi // BioMetals. 2013. Vol. 26, N 5. P. 813-825.

15. Shulman A., Laycock G. A. Action of 1,10-phenanthroline transition metal chelates on P388 mouse lymphocyte leukaemic cells // Chem.-Biol. Interactions. 1977. Vol. 16, N 1. P. 89-99.

16. Пастон С. В., Бакулев В. М., Демидов В. Н., Николаев А. И., Касъяненко Н. А. Исследование взаимодействия ДНК с новым 1,10-фенантроцианиновым комплексом цинка спектральными методами // Вестник СПбГУ. Серия 4. Физика. Химия. 2015. Т. 2 (60), вып. 3. С. 299-304.

17. Демидов В. Н. Электрон-избыточные 1,10-фенантроцианиновые комплексы d-элементов: закономерности образования, спектральные свойства, структурно-термодинамическое подобие: дис. ... д-ра хим. наук. Санкт-Петербургский государственный технологический институт (технический университет), 2010. 481 с.

18. Yi H., Jutand A., Lei A. Evidence for the interaction between ${ }^{t} \mathrm{BuOK}$ and 1,10 -phenanthroline to form the 1,10-phenanthroline radical anion: a key step for the activation of aryl bromides by electron transfer // Chem. Commun. 2015. Vol. 51. P. 545-548.

19. Trabolsi A., Khashab N., Fahrenbach A.C., Friedman D.C., Colvin M.T., CotíK. K., BenítezD., TkatchoukE., Olsen J.-C., Belowich M.E., CarmielliR., Khatib H.A., Goddard W.A., Wasielewski M. R., Stoddart J. F. Radically enhanced molecular recognition // Nature Chem. 2010. Vol. 2. P. $42-49$.

20. Панина Н. С., Демидов В. Н., Симанова С. А. Исследование DFT 2,2'-би-1,10-фенантролина и его восстановленной формы как потенциального лиганда для новых тетраазахромофорных комплексов // Журн. общей химии. 2008. Т. 78, № 5. С. 771-777.

21. Калнинъш K. K. Перенос водорода в органической химии. Издат.-полиграф. центр СПГУТД, 2012. 417 c.

\section{References}

1. Oladipo M.A., Olaoye O. J. Antimicrobial, DNA cleavage and antitumoral properties of some transition metal complexes of 1,10-phenanthroline and 2,2'-bi-pyridine: A review. Int. J. Res. Pharm. Biomed. Sci., 2013, vol. 4, no 4, pp. 1160-1171.

2. Mahalakshmi R., Raman N. A therapeutic journey of mixed ligand complexes containing 1,10-phenanthroline derivatives: a review. Int. J. Curr. Pharm. Res., 2016, vol. 8, no 3, pp. 1-6.

3. Shulman A., White D. O. Virostatic activity of 1,10-phenanthroline transition metal chelates: A structure-activity analysis. Chem.-Biol. Interactions, 1973, vol. 6, pp. 407-413.

4. Dwyer F. P., Reid I. K., Shulman A., Laycock G. M., Dixson S. The biological actions of 1,10-phenanthroline and 2, $2^{\prime}$-bipyridine hydrochlorides, quaternary salts and metal chelates and related compounds. 
1. Bacteriostatic action on selected gram-positive, gram-negative and acid-fast bacteria. Austral. J. Exper. Biol. Med. Sci., 1969, vol. 47, pp. 203-218.

5. Shulman A., Cade G., Dumble L., Laycock G. M. The lethal action of 1,10-phenanthroline transition metal chelates and related compounds on dermatophytes and Candida albicans. Arzneimittelforschung, 1972, vol. 22, no 1, pp. 154-158.

6. Barton J. K., Dannenberg J. J., Raphael A. L. Enantiomeric selectivity in binding tris-(phenanthroline)zinc(II) to DNA. J. Amer. Chem. Soc., 1982, vol. 104, no 18, pp. 4967-4969.

7. Shahabadi N., Mohammadi S. Synthesis, characterization and DNA interaction studies of a new Zn(II) complex containing different dinitrogen aromatic ligands. Bioinorg. Chem. Appl., 2012, vol. 2012. 571913.

8. Anjomshoa M., Torkzadeh-Mahani M., Shakeri M. The Zn(II) nanocomplex: sonochemical synthesis, characterization, DNA- and BSA-binding, cell imaging, and cytotoxicity against the human carcinoma cell lines. J. Fluorescence, 2016, vol. 26, no 3, pp. 1007-1020.

9. Yuan Cai-Xia, Wei Yi-Bin, Yang Pin. DNA-binding and cleavage studies of zinc(II) mixed-polypyridyl complex. Chinese J. Chem., 2006, vol. 24, no 8, pp. 1006-1012.

10. Hoi-Ling Seng, Sze-Tin Von, Kong-Wai Tan, Mohd Jamil Maah, Seik-Weng Ng, Raja Noor Zaliha Raja Abd Rahman, Ignez Caracelli, Chew-Hee Ng. Crystal structure, DNA binding studies, nucleolytic property and topoisomerase I inhibition of zinc complex with 1,10-phenanthroline and 3-methyl-picolinic acid. BioMetals, 2010, vol. 23, pp. 99-118.

11. Von S.-T., Seng H.-L., Lee H.-B., Ng S.-W., Kitamura Y., Chikira M., Ng C.-H. DNA molecular recognition and cellular selectivity of anticancer metal(II) complexes of ethylene- diaminediacetate and phenanthroline: multiple targets. J. Biolog. Inorg. Chem., 2012, vol. 17, no 1, pp. 57-69.

12. Seng H.-L., Ong H.-K.A., Rahman R. N. Z. R. Abd., Yamin B. M., Tiekink E. R. T., Tan K.W., Maah M. J., Caracelli I., Ng C.-H. Factors affecting nucleolytic efficiency of some ternary metal complexes with DNA binding and recognition domains. Crystal and molecular structure of Zn(phen)(edda). J. Inorg. Biochem., 2008, vol. 102, no 11, pp. 1997-2011.

13. Qin Wang, Min Huang, Yu Huang, Jin-Sheng Zhang, Gui-Feng Zhou, Ren-Quan Zeng, Xin-Bin Yang. Synthesis, characterization, DNA interaction, and antitumor activities of mixed- ligand metal complexes of kaempferol and 1,10-phenanthroline/2, 2'-bipyridine. Med. Chem. Res., 2014, vol. 23, no 5, pp. 2659-2666.

14. Gouvea L. R., Martins D. A., Batista D. G. J., Soeiro M. N. M. C., Louro S. R. W., Barbeira P. J. S., Teixeira L. R. Norfloxacin Zn(II)-based complexes: acid base ionization constant determination, DNA and albumin binding properties and the biological effect against Trypanosoma cruzi. BioMetals, 2013, vol. 26, no 5, pp. 813-825.

15. Shulman A., Laycock G. A. Action of 1,10-phenanthroline transition metal chelates on P388 mouse lymphocyte leukaemic cells. Chem.-Biol. Interactions, 1977, vol. 16, no 1, pp. 89-99.

16. Paston S. V., Bakulev V. M., Demidov V. N., Nikolaev A. I., Kasyanenko N. A. Issledovanye vzaimodeystvia DNK s novym 1,10-fenantrotsianinovym kompleksom tsinka. [Study of DNA interaction with new 1,10-phenanthrocyanine Zn complexes by spectral methods]. Vestnik St. Petersburg University. Series 4. Physics. Chemistry, 2015, vol. 2 (60), iss. 3, pp. 299-304. (In Russian)

17. Demidov V. N. Elektronno-izbytochnye 1,10-fenantrotsianinovye kompleksy d-elementov: zakonomernosti obrazovaniia, spektral'nye svoistva, strukturno-termodinamicheskoe podobie [Electron-rich 1,10phenantrocyanine complexes of d-elements: regularities of formation, spectral properties, structural-thermodynamic similarity. Dr. Chem. Sci. thesis]. St. Petersburg state institute of technology (technical university), 2010. 481 p.

18. Yi H., Jutand A., Lei A. Evidence for the interaction between ${ }^{t} \mathrm{BuOK}$ and 1,10 -phenanthroline to form the 1,10-phenanthroline radical anion: a key step for the activation of aryl bromides by electron transfer. Chem. Commun., 2015, vol. 51, pp. 545-548.

19. Trabolsi A., Khashab N., Fahrenbach A. C., Friedman D.C., Colvin M. T., CotíK. K., Benítez D., Tkatchouk E., Olsen J.-C., Belowich M. E., Carmielli R., Khatib H. A., Goddard W. A., Wasielewski M. R., Stoddart J. F. Radically enhanced molecular recognition. Nature Chem., 2010, vol. 2, pp. 42-49.

20. Panina N. S., Demidov V. N., Simanova S. A. Issledovanye DFT 2,2'-bi-1,10-fenantrolina i ego vosstanovlennoy formy kak potentsialnogo liganda dlya novykh tetraazakhro-mofornykh kompleksov [A DFT study of 2,2'-bi-1,10-phenanthroline and its reduced form as a potential ligand for new tetraaza chromophore complexes]. Zhurn. obshchei khimii. [Rus. J. Gen. Chem.], 2008, vol. 78, no 5, pp. 771-777. (In Russian)

21. Kalnynsh K. K. Perenos vodoroda v organitscheskoy khimii [Hydrogen transfer in organic chemistry]. Ed.-poligraph. center SPGUTD, 2012. 417 p. (In Russian)

Статья поступила в редакцию 30 июня 2016 г. 
Контактная информация

Демидов Виктор Николаевич - доктор химических наук, доцент; e-mail: vndemidov@mail.ru

Сухаржевский Станислав Михайлович - кандидат геолого-минералогических наук; e-mail: stanislav.sukharzhevskii@spbu.ru

Пастон Софъя Владимировна - кандидат физико-математических наук, доцент;

e-mail: svpaston@list.ru

Зинченко Андрей Викторович - кандидат химических наук, доцент; e-mail: zinch@mail.ru

Веденеева Лидия Николаевна - кандидат химических наук, доцент; e-mail: vedeneeva@bf.pstu.ru

Пахомова Татъяна Борисовна - кандидат химических наук, доцент; e-mail: pakhom@list.ru

Demidov Viktor N. - Doctor of Chemistry, Associate Professor; e-mail: vndemidov@mail.ru

Sukharzhevsky Stanislav M. — PhD; e-mail: stanislav.sukharzhevskii@spbu.ru

Paston Sofia V. — PhD, Associate Professor; e-mail: svpaston@list.ru

Zinchenko Andrey V. — PhD, Associate Professor; e-mail: zinch@mail.ru

Vedeneeva Lidya N. — PhD, Associate Professor; e-mail: vedeneeva@bf.pstu.ru

Pakhomova Tatyana B. - PhD, Associate Professor; e-mail: pakhom@list.ru 